\title{
AUTHOR INDEX, VOLUME 31, 1994
}

Adams, R. A., 603
Agarwal, M., 11
Ando, J., 57
Antaki, J. F., 297
Bai, J.J., 77
Ballyk, P. D., 565
Barbenel, J. C., 193
Biviji, A., 511
Bjelle, A., 407
Bjursten, L. M., 617
Blackwell, J., 21
Bleuit, J., 163
Borovetz, H. S., 297
Braide, M., 617
Buchsbaum, G., 327,339
Chen, D., 103
Chen, J. H., 163
Chien, S., 77
Cobbold, R. S. C., 115
Dao, K. M., 69
Delfino, A., 533
Deligianni, D. D., 245
Deng, L. H., 193
Dhall, D. P., 259
Dobashi, T., 383
Ebrahimzadeh, P. R., 617
Ethier, C. R., 565
Evans, S.-A., 603
Freedman, J. C., 91
Friederichs, E., 207
Gudmundsson, M., 407
Hakim, T. S., 91
Ham, A., 163
Hashimoto, S., 521
Hayashi, K., 365
Hoson, T., 171
Hsu, S., 21
Hwang, N. H. C., 549
Iannotti, J. P., 163
Idonuma, A., 383

Ishikawa, C., 57

Izumida, Y., 395

Jamieson, A. M., 21

Johnson, A. E., 69

Jones, J. G., 603

Kabat, E. A., 353

Kafka, V., 245

Kameneva, M. V., 297

Kamisaka, S., 171

Kamiya, A., 57

Kaniewski, W. S., 91

Karpova, E. V., 221

Katayama, Y., 57

Kaul, D. K., 103

King, M., 11, 511

Kobayashi, Y., 235

Korenaga, R., 57

Kormos, R. L., 297

Kuo, C.-D., 77

Lee, B., 327, 339

Litt, M., 327, 339

Long, M., 287

Lowe, G. D. O., 193

Maeda, N., 277, 395

Masuda, Y., 171

Matsumoto, T., 365

Meiselman, H. J., 207

Meister, J. J., 533

Menzel, E.J., 37

Miftakhov, R., 309

Missirlis, Y. F., 245

Mo, L. Y.-L., 115

Moore Jr., J. E., 533

Muralidharan, E., 277, 587

Naiki, T., 365

Nair, C. H., 259

Nakajima, T., 395

Nash, G. B., 643

Nieuw Amerongen, A. V., 631

Nishinari, K., 171, 235
O'Rear, E. A., 69

Odén, A., 407

Ohshima, N., 143

Ohtsuka, A., 57

Okamoto, A., 235

Okano, M., 155

Osipova, T. N., 221

Paulitschke, M., 643

Peitersen, S. E., 69

Preece, A., 643

Reihsner, R., 37

Sakanishi, A., 287, 383

Sato, M., 143

Seiyama, A., 395

Shehada, R. E. N., 115

Shukla, J. B., 11

Steinman, D. A., 565

Strid, K.-G., 617

Sugihara-Seki, M., 1

Sung, L. A., 353

Suzuki, Y., 395

Tateishi, N., 277, 395

Thurston, G. B., 179

Tomkiewicz, R. P., 511

Toyama, Y., 383

Van der Reijden, W. A., 631

van Gelder, J. M., 259

Veerman, E. C. I., 631

Vorob'ev, V. I., 221

Wang, H. B., 287

Wang, S. K, 549

Watach, M. J., 297

Williamns, J. !.., 163

Wingate, D., 309

Wu, Y. P., 287

Wu, Z. Z., 287

Yamamoto, R., 171

Yoshida, Y., 155 